\title{
Sidelobe Suppression with Null Steering by Independent Weight Control
}

\author{
Zafar-Ullah Khan, ${ }^{1}$ Aqdas Naveed Malik, ${ }^{1}$ Fawad Zaman, ${ }^{2}$ \\ Syed Azmat Hussain, ${ }^{3}$ and Abdul-Rehman Khan ${ }^{2}$ \\ ${ }^{1}$ Department of Electronic Engineering, IIU, H-10, Islamabad 44000, Pakistan \\ ${ }^{2}$ Department of Electrical Engineering, COMSATS Institute of Information Technology, Attock Campus, Attock 43600, Pakistan \\ ${ }^{3}$ School of Engineering and Applied Sciences, ISRA University, Islamabad Campus, Islamabad 44000, Pakistan \\ Correspondence should be addressed to Zafar-Ullah Khan; zafarullah.phdee13@iiu.edu.pk
}

Received 20 April 2015; Accepted 18 May 2015

Academic Editor: Vincenzo Galdi

Copyright (C) 2015 Zafar-Ullah Khan et al. This is an open access article distributed under the Creative Commons Attribution License, which permits unrestricted use, distribution, and reproduction in any medium, provided the original work is properly cited.

\begin{abstract}
A uniform linear array of $n$ antenna elements can steer up to $n-1$ nulls. In situations where less than $n-1$ nulls are required to be steered, the existing algorithms have no criterion to utilize the remaining weights for sidelobe suppression. This work combines sidelobe suppression capability with null steering by independent weight control. For this purpose, the array factor is transformed as the product of two polynomials. One of the polynomials is used for null steering by independent weight control, while the second one is for sidelobe suppression whose coefficients or weights are determined by using convex optimization. Finally, a new structure is proposed to incorporate the product of two polynomials such that sidelobe suppression weights are decoupled from those of null steering weights. Simulation results validate the effectiveness of the proposed scheme.
\end{abstract}

\section{Introduction}

Null steering for adaptive beamforming is an important area of research due to its military and commercial applications [1-6]. Null steering techniques can broadly be classified as Direction of Arrival (DOA) based beamformers and optimal adaptive beamformers [1]. Existing DOA based null steering techniques either control the excitation of elements' amplitude only $[2,3]$, phase only $[4,5]$, or both $[6]$. There are also techniques that achieve null steering by changing the position and/or elevations of the array elements [7-10].

Elements' phase and amplitude excitation techniques are slow for large arrays because even if a single null changes its position, the whole set of weights is required to be reevaluated which is obviously time consuming and complicated. On the other hand, null steering by controlling the excitation amplitude only is easy to implement and less sensitive to quantization error but reduces the number of steerable nulls $[2,3]$. The techniques like phase only and position perturbations of the array elements are nonlinear problems and cannot be solved directly by analytical methods [11]. Besides, position perturbations methods require servo motors for the movement of the elements and, in case of large arrays, the complexity to control element position increases due to increase in computational time to find new position perturbations.

The solution for this problem is the null steering by independent weight control where if a single null changes its position, only the weight set corresponding to that null is evaluated and changed [3,6]. Unfortunately, these DOA based beamformers do not have sidelobe suppression capability. Although this problem has been addressed for optimal adaptive beamforming algorithms $[12,13]$ the method cannot be used in DOA based beamformers because in these algorithms pattern synthesis weights are not determined by using optimization techniques.

This paper presents a technique where the array factor is transformed as product of two polynomials such that one polynomial denoted by $\mathrm{AF}_{1}$ provides independent steering of all available nulls while the other denoted by $\mathrm{AF}_{2}$ suppresses 
sidelobes by utilizing remaining weights. The coefficients of $\mathrm{AF}_{1}$ are determined by forcing the zeros of $\mathrm{AF}_{1}$ to lie on unit circle in the complex plane [6] while the coefficients of $\mathrm{AF}_{2}$ are evaluated using convex optimization. Furthermore a structure is presented to implement this array factor where the sidelobe suppression weights are decoupled from the weights meant for independent null steering. Therefore the coefficients of $\mathrm{AF}_{2}$ will not be required to change with the change in position of any interference.

The rest of the work is organized as follows. Section 2 presents problem formulation, while proposed technique and structure are discussed in Sections 3 and 4, respectively. Simulation results are given in Section 5. Finally, conclusion and future work recommendation are given in Section 6.

\section{Problem Formulation}

Consider a uniform linear array (ULA) of $N$ omnidirectional elements. Let $d$ be the element spacing, $\alpha$ the progressive phase, and $\theta$ the angle of arrival of the plane wave impinging on the array. Figure 1 shows the path difference of this wave on the adjacent elements of the ULA; that is, $x=d \cos \theta$. The output of the array elements is multiplied by the properly selected coefficients $A_{0}, A_{1}, \ldots, A_{N-1}$ to steer the nulls in the directions of interferences. We take the first element (element $0)$ of the array as reference and $\psi=\alpha+(2 \pi / \lambda) d \cos \theta$, where $k=2 \pi / \lambda$ is known as wave number. The signal at element 2 is the delayed version of the signal at element 1 and is expressed as $e^{j \psi}$. Let $e^{j \psi}=z$; then the array factor can be given as

$$
\mathrm{AF}_{1}=A_{0}+A_{1} z+\cdots+A_{N-1} z^{(N-1)}
$$

This $\mathrm{AF}_{1}$ is the $N-1$-degree polynomial and has $N-1$ roots (nulls) dependent on coefficients $A_{0}, A_{1}, \ldots, A_{N-1}$. In factorized form, (1) becomes

$$
\mathrm{AF}_{1}=\left(z-z_{1}\right)\left(z-z_{2}\right) \cdots\left(z-z_{N-1}\right)
$$

where $z_{1}, z_{2}, \ldots, z_{N-1}$ are zeros forced to exist on the unit circle in the complex plane $[2,3,6,14]$ and their position on the circle depends on coefficients $A_{0}, A_{1}, \ldots, A_{N-1}$.

To steer the main beam in the desired direction $\theta_{s}$, the progressive phase shift is given by

$$
\alpha=-\left(\frac{2 \pi}{\lambda}\right) d \cos \theta_{s} .
$$

In order to incorporate expression (1), factors of (2) are to be multiplied iteratively by combining the coefficients of the same powers of $z$ at each step. This process of multiplication is complicated and time consuming for large arrays. The algorithms proposed in $[3,6]$ avoid this multiplication by decoupling null steering weights, while the current work presents a method to add sidelobe suppression capability to these algorithms by adjusting and decoupling the weights of spare elements of the array from those of null steering weights.

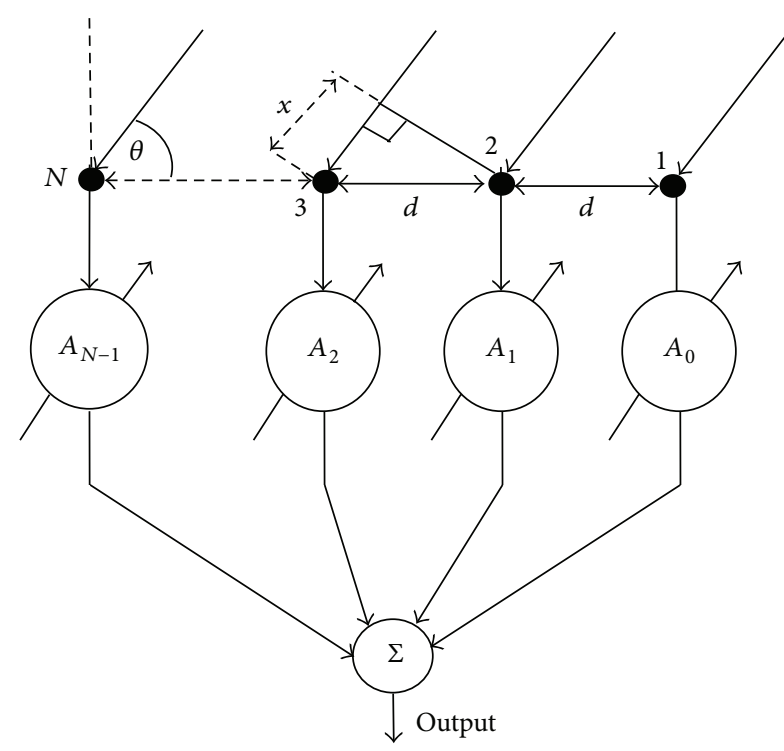

FIgURE 1: Uniform linear array of $N$ elements with $N-1$ steerable nulls.

\section{Proposed Algorithm for Independent Null Steering (INS) with Suppressed Sidelobes}

According to Schelkunoff [14], the product of two or more polynomials results in a polynomial and there exists a linear array corresponding to this resultant polynomial. Using the same fact, consider the desired array factor AF given below:

$$
\mathrm{AF}=\mathrm{AF}_{1} \cdot \mathrm{AF}_{2},
$$

where $\mathrm{AF}_{1}$ is meant for independent steering of $N_{1}$ nulls and can be given as

$$
\mathrm{AF}_{1}=\prod_{i=1}^{N_{1}}\left(z-z_{i}\right)
$$

The purpose of $\mathrm{AF}_{2}$ is to suppress sidelobes and it can be expressed by

$$
\mathrm{AF}_{2}=b_{0}+b_{1} z+\cdots+b_{N_{2}-1} z^{N_{2}-1}=\mathbf{s}_{N_{2}} \mathbf{b}
$$

where $\mathbf{b}=\left[b_{0}, b_{1}, \ldots, b_{N_{2}-1}\right]^{T}$ represents the complex weight vector and $\mathbf{s}_{\mathrm{N}_{2}}=\left[1, z, \ldots, z^{\mathrm{N}_{2}-1}\right]$ is the steering vector. The condition on the vector $\mathbf{b}$ is that it guarantees the sidelobe suppression and steers the main beam in the same direction as that of $\mathrm{AF}_{1}$. To find out $\mathbf{b}$, consider a set $S_{R}$ that is union of two sets containing angles from the left and right sidelobe regions of the main beam with beam-width $\theta_{\mathrm{mb}}$; that is,

$$
S_{R}=\left\{0<\theta \leq \theta_{s}-\frac{\theta_{\mathrm{mb}}}{2}\right\} \cup\left\{\theta_{s}+\frac{\theta_{\mathrm{mb}}}{2}<\theta \leq 180^{\circ}\right\} .
$$

Then $p$ discrete angles are selected from $S_{R}$ and placed in another set $A_{S_{R}}$ given by

$$
A_{S_{R}}=\left\{\theta_{i} \mid \theta_{i} \in S_{R} \wedge \theta_{i}=\theta_{0}+i \delta \theta\right\} \subseteq S_{R},
$$




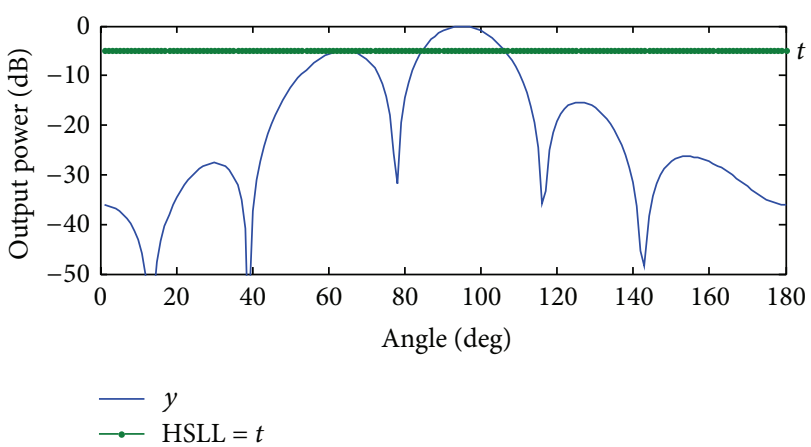

(a)

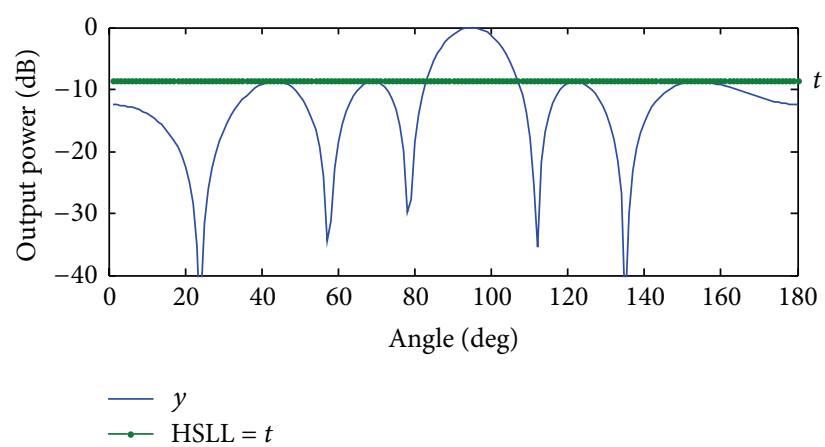

(b)

Figure 2: (a) Highest sidelobe level (HSLL $=t$ ) is required to be minimized when $N_{2}=6, \theta_{s}=95^{\circ}$; (b) $y=\mathrm{AF}_{2}$ pattern when highest sidelobe level (HSLL $=t$ ) has been minimized for $N_{2}=6, \theta_{s}=95^{\circ}$, and $\theta_{\mathrm{mb}}=24^{\circ}$.

where $i=0,1, \ldots, p-1$ and $\theta_{0}$ is the starting angle and $\delta \theta$ is the step size.

Now consider another matrix A, containing steering vectors corresponding to the angles contained in $A_{S_{R}}$; that is,

$$
\mathbf{A}=\left[\mathbf{s}_{N_{2}}\left(\theta_{0}\right) \mathbf{s}_{N_{2}}\left(\theta_{1}\right) \cdots \mathbf{s}_{N_{2}}\left(\theta_{p-1}\right)\right]^{T},
$$

where $\mathbf{s}_{N_{2}}\left(\theta_{i}\right)=\left[1, z_{i}, \ldots, z_{i}^{N_{2}-1}\right]$ with $z_{i}=\exp (j(\alpha+$ $\left.\left.(2 \pi / \lambda) d \cos \theta_{i}\right)\right)$. Now the requirement is to minimize the array output power along the angles $\theta_{i} \in A_{S_{R}}$ subject to the unit output along the desired direction $\theta_{s}$.

The requirement is fulfilled if we restrict the peak output power in the sidelobe region, that is, the highest sidelobe level (HSLL) to have minimum value as shown in Figure 2. Minimizing this power along the angles $\theta_{i} \in A_{S_{R}}$ is the same as minimizing the absolute output along these angles; that is,

$$
\min _{\mathbf{b}} \max \left(\left|\mathbf{s}_{N_{2}}\left(\theta_{i}\right) \mathbf{b}\right|\right), \quad \theta_{i} \in A_{S_{R}}
$$

subject to $\mathbf{s}_{\mathrm{N}_{2}}\left(\theta_{s}\right) \mathbf{b}=1$.

Since the steering vectors along these angles are contained in matrix $\mathbf{A}$ the absolute output along these angles is the vector $|\mathbf{A b}|$ and the above minimization problem becomes

$$
\begin{array}{cl}
\min _{\mathbf{b}} & \max |\mathbf{A b}| \\
\text { subject to } & \mathbf{s}_{\mathrm{N}_{2}}\left(\boldsymbol{\theta}_{s}\right) \mathbf{b}=1 .
\end{array}
$$

In (11) the objective function controls the sidelobe level and the constraint forms the main beam in the desired direction. This problem can be cast as the second-order cone program in the following manner:

$$
\begin{array}{ll}
\text { Minimize } & t \\
\text { subject to } & |\mathbf{A} \mathbf{b}| \leq t \\
& \mathbf{s}_{\mathrm{N}_{2}}\left(\theta_{s}\right) \mathbf{b}=1 .
\end{array}
$$

The inequalities in (12) are called second-order cone constraints and $(\mathbf{A b}, t) \in$ second-order cone in $\mathbf{R}^{p+1}[15]$. Secondorder cone program is the subclass of convex optimization and hence problem (11) can be solved using [16].
The polynomial $\mathrm{AF}_{2}$ suppresses sidelobe levels and has overlapping main beam with that of $\mathrm{AF}_{1}$. Due to pattern multiplication, $\mathrm{AF}=\mathrm{AF}_{1} \cdot \mathrm{AF}_{2}$ will give the independent null steering capability with suppressed sidelobes. The $\mathrm{AF}_{2}$ is required to be calculated once and remains unchanged as long as the position of the main beam is unchanged. However if the position of the main beam is changed, the coefficients of both the polynomials, that is, $\mathrm{AF}_{1}$ and $\mathrm{AF}_{2}$, will have to be recalculated and changed.

\section{Proposed Structure}

In this section, a structure is proposed to steer 5 nulls independently and to use three weights for sidelobe suppression; that is, $N_{1}=5, N_{2}=3$. This structure can easily be extended for any values of $N_{1}$ and $N_{2}$. The structure starts with ULA of 8 elements, that is, $\left(N_{1}+N_{2}\right)$. It can be seen from Figure 3 that, for first $N_{1}$ (which is 5 in this case) stages, each adder has 2 inputs. In the structure, first stage controls the position of first null where first input of each adder is multiplied by $\left(-z_{1}\right)$. The $j$ th output of this stage is represented by $y_{1, j}$ with $j=1, \ldots, N_{1}+N_{2}-1$ and is given by

$$
y_{1, j}=z^{j-1}\left(z-z_{1}\right) \quad \text { for } j=1, \ldots, N_{1}+N_{2}-1 .
$$

Similarly second stage controls second null by multiplying first input of each of its adders by $\left(-z_{2}\right)$. These outputs are

$$
\begin{aligned}
& y_{2, j}=z^{j-1}\left(z-z_{1}\right)\left(z-z_{2}\right) \\
& \qquad \text { for } j=1, \ldots, N_{1}+N_{2}-2 .
\end{aligned}
$$

It is clear from Figure 3 that we will get $N_{2}$ outputs after $N_{1}$ stages and these outputs will be

$$
\begin{aligned}
y_{N_{1}, j}=z^{j-1} \prod_{i=1}^{N_{1}}\left(z-z_{i}\right)=z^{j-1} \mathrm{AF}_{1} & \\
& \text { for } j=1, \ldots, N_{2} .
\end{aligned}
$$

It means these outputs are multiples of $\mathrm{AF}_{1}$ as shown below:

$$
\left[y_{N_{1}, 1}, y_{N_{1}, 2}, \ldots, y_{N_{1}, N_{2}}\right]^{T}=\operatorname{AF}_{1}\left[1, z, \ldots, z^{N_{2}-1}\right]^{T}
$$




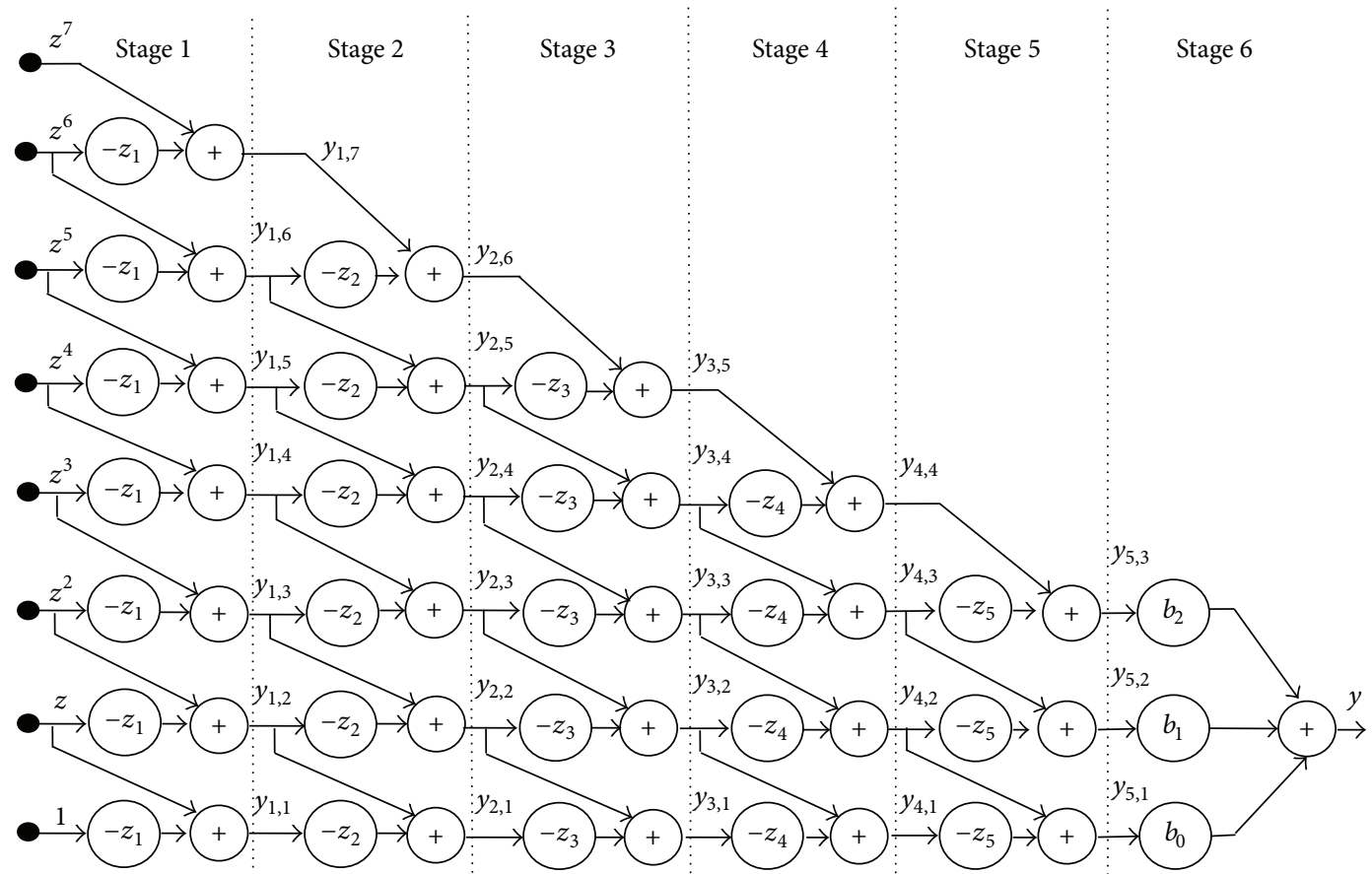

FIGURE 3: Structure for independent null steering with suppressed sidelobes.

Thus first $N_{1}$ stages will incorporate $\mathrm{AF}_{1}$ and steer $N_{1}$ nulls by independent weight control. After that the resulting $N_{2}$ outputs are weighted to give $\mathrm{AF}_{2}$ for sidelobe suppression. Finally these weighted outputs are summed to result in $\mathrm{AF}=$ $\mathrm{AF}_{1} \cdot \mathrm{AF}_{2}$ given by (17) and the corresponding structure is shown in Figure 3. Consider

$$
\begin{aligned}
y & =\mathrm{AF}_{1}\left[1, z, \ldots, z^{N_{2}-1}\right]\left[b_{0}, b_{1}, \ldots, b_{N_{2}-1}\right]^{T} \\
& =\mathrm{AF}_{1} \mathrm{AF}_{2} .
\end{aligned}
$$

As mentioned earlier, convex optimization is used to find out the $N_{2}$ complex weights. These $N_{2}$ weights remain unchanged even if one or more nulls change their position. On the other hand, if the main beam changes its position, the whole set of weights, including INS weights for $N_{1}$ stages and $N_{2}$ weights for sidelobe control, is changed. Even in such situation implementation of $\mathrm{AF}_{1}$ in Figure 3 is not time consuming as compared to Figure 1, because it incorporates factors directly without multiplication and forming a single polynomial.

\section{Simulation and Results}

In this section, several simulation examples are carried out in MATLAB to verify the effectiveness of the proposed algorithm. In these examples element spacing $d$ is taken as $\lambda / 2$.

Example 1. This example compares the performance of the proposed technique with two algorithms, that is, INS by decoupling real weights (Realwt) and INS by decoupling complex weights (Cmplxwt). For this purpose, main beam is taken along $95^{\circ}$, while the four interferences are placed at $20^{\circ}$, $70^{\circ}, 120^{\circ}$, and $160^{\circ}$. In this case, INS by decoupling real weights and INS by decoupling complex weights require ULAs having 9 and 5 elements, respectively, while the proposed algorithm is utilized using a ULA of 9 elements with $N_{1}=4$ and $N_{2}=5$. One can clearly see in Figure 4 that the performance of the proposed algorithm is better for sidelobe suppression as compared to the other two algorithms.

Example 2. This example compares the performance of the proposed algorithm with INS by decoupling real weights when the second null is at $70^{\circ}$ and also when it is shifted to $75^{\circ}$, while positions of all the other nulls remain unchanged. In Figure 5, again one can see that the proposed scheme outperforms Realwt, no matter whether the second null is at $70^{\circ}$ or is shifted to $75^{\circ}$. Similar behavior can be observed if the position of multiple nulls is changed.

Example 3. This example demonstrates the effect of number of coefficients of $\mathrm{AF}_{2}$, that is, $\mathrm{N}_{2}$ on the sidelobe level. For this purpose sidelobe levels are compared for the same set of signals as in Example 1, when $N_{2}=4,6$, and 8. It is clear from Figure 6 that the sidelobe suppression capability of the proposed algorithm increases as $N_{2}$ is increased.

Example 4. This example shows the capability of the proposed algorithm to decouple complex weights of $\mathrm{AF}_{1}$ to steer nulls independently. For this purpose main beam is taken along $95^{\circ}$ and four interferences are placed at $20^{\circ}, 70^{\circ}$, $120^{\circ}$, and $160^{\circ}$. Then the position of interferences is changed one by one and the results are shown in Table 1 where the changed position of the nulls and corresponding weights are shown by bold entries. It can easily be deduced that when any interference changes its position, only the corresponding weight is changed, while the rest of the weights remain unaffected. 
TABLE 1: Decoupling of weights by proposed algorithm for INS when $\theta_{s}=95^{\circ}, N_{1}=4, N_{2}=5$.

\begin{tabular}{|c|c|c|c|c|c|}
\hline S. number & $\theta_{i}$ & $z_{1}$ & $z_{2}$ & $z_{3}$ & $z_{4}$ \\
\hline 1 & $20^{\circ}, 70^{\circ}, 120^{\circ}, 160^{\circ}$ & $-0.9964-0.0842 i$ & $0.2207+0.9753 i$ & $0.2704-0.9627 i$ & $-0.8946-0.4469 i$ \\
\hline 2 & $25^{\circ}, 70^{\circ}, 120^{\circ}, 160^{\circ}$ & $-0.9998+0.0205 i$ & $0.2207+0.9753 i$ & $0.2704-0.9627 i$ & $-0.8946-0.4469 i$ \\
\hline 3 & $20^{\circ}, 65^{\circ}, 120^{\circ}, 160^{\circ}$ & $-0.9964-0.0842 i$ & $-0.0307+0.9995 i$ & $0.2704-0.9627 i$ & $-0.8946-0.4469 i$ \\
\hline 4 & $20^{\circ}, 70^{\circ}, 125^{\circ}, 160^{\circ}$ & $-0.9964-0.0842 i$ & $0.2207+0.9753 i$ & $0.0426-0.9991 i$ & $-0.8946-0.4469 i$ \\
\hline 5 & $20^{\circ}, 70^{\circ}, 120^{\circ}, 155^{\circ}$ & $-0.9964-0.0842 i$ & $0.2207+0.9753 i$ & $0.2704-0.9627 i$ & $-0.8429-0.5381 \mathrm{i}$ \\
\hline
\end{tabular}

TABLE 2: Effect of change in $\theta_{s}$ on different parameters.

\begin{tabular}{|c|c|c|c|}
\hline S. number & $\theta_{s}$ & Parameters & Value \\
\hline \multirow{3}{*}{1} & \multirow{3}{*}{$95^{\circ}$} & $b_{0}-b_{4}$ & $0.0989+0.0098 i, 0.2426+0.0120 i, 0.3171,0.2426-0.0120 i, 0.0989-0.0098 i$ \\
\hline & & $z_{1}-z_{4}$ & $-0.9964-0.0842 i, 0.2207+0.9753 i, 0.2704-0.9627 i,-0.8946-0.4469 i$ \\
\hline & & $\alpha$ & 0.2738 \\
\hline \multirow{3}{*}{2} & \multirow{3}{*}{$90^{\circ}$} & $b_{0}-b_{4}$ & $0.0987,0.2427,0.3172,0.2427,0.0987$ \\
\hline & & $z_{1}-z_{4}$ & $-0.9821-0.1883 i, 0.4762+0.8793 i, 0-i,-0.9821-0.1883 i$ \\
\hline & & $\alpha$ & 0 \\
\hline
\end{tabular}

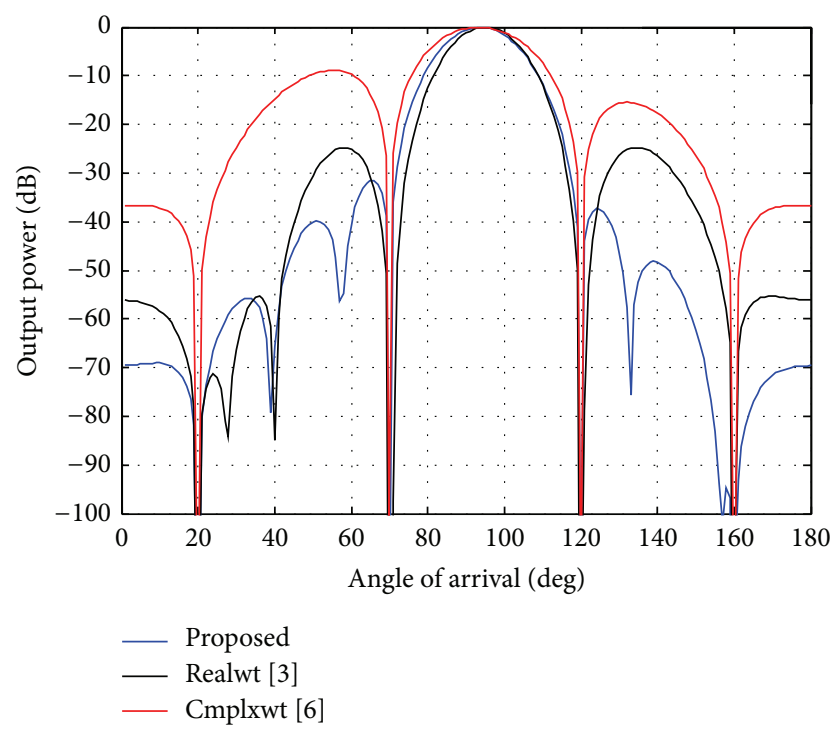

FIGURE 4: Performance comparison of the proposed algorithm with INS by decoupling real weights (Realwt) and INS by decoupling complex weights (Cmplxwt).

Example 5. This example shows the effect of change in direction of main beam on $z_{i}, \mathbf{b}$, and $\alpha$. For this purpose initially main beam is taken along $95^{\circ}$ while four interferences are placed at $20^{\circ}, 70^{\circ}, 120^{\circ}$, and $160^{\circ}$. Then main beam is shifted at $90^{\circ}$ while the interferences do not change their position as given in Table 2 for both the cases. It is clear that these parameters change with the change in $\theta_{s}$.

\section{Conclusion}

A method for uniform linear array is proposed to steer the nulls by independent weight control and to suppress sidelobes. It uses the progressive phase shift for beam steering and product of two polynomials for independent null steering

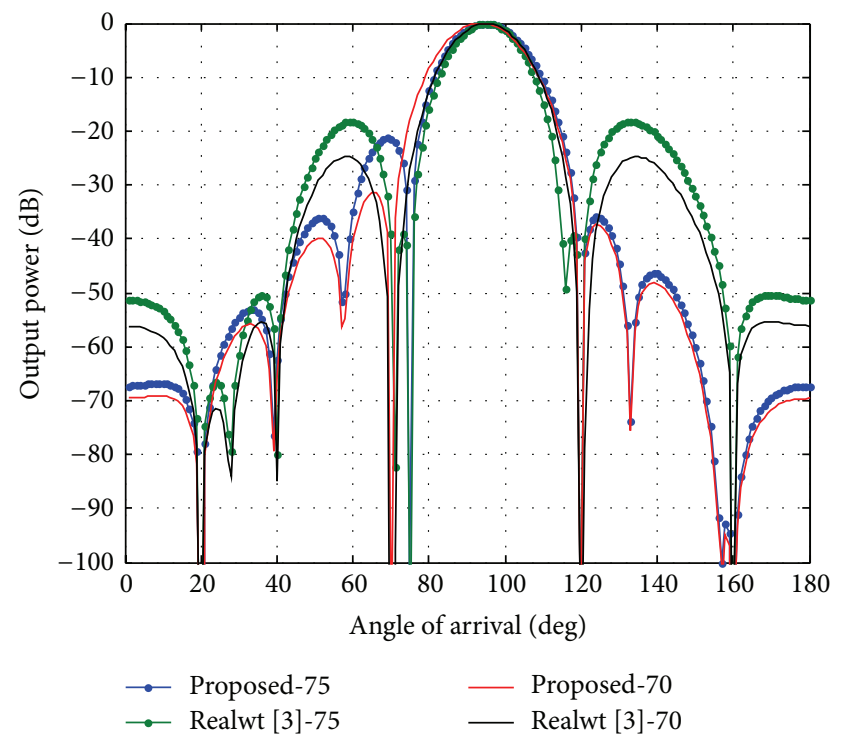

FIGURE 5: Performance comparison of proposed algorithm with INS by decoupling real weights [3], when second null changes its position from $70^{\circ}$ to $75^{\circ}$.

with sidelobe suppression. The zeros of independent null steering polynomial are forced to lie on the unit circle in the complex plane. Similarly, the coefficients for sidelobe suppression are determined by convex optimization. Then a structure is proposed that incorporates the product of the two polynomials. In order to steer $N_{1}$ nulls independently and to use $N_{2}$ weights for sidelobe suppression, a ULA of $N_{1}+N_{2}$ elements is required. In the structure, the number of weights for independent null steering and the total number of weights will be $0.5 N_{1}\left(N_{1}+2 N_{2}-1\right)$ and $0.5 N_{1}\left(N_{1}+2 N_{2}-1\right)+N_{2}$, respectively. Traditionally this array can steer $N_{1}+N_{2}-1$ nulls but the proposed technique steers only $N_{1}$ nulls with suppressed sidelobes at the cost of $0.5 N_{1}\left(N_{1}+2 N_{2}-1\right)+N_{2}$ 


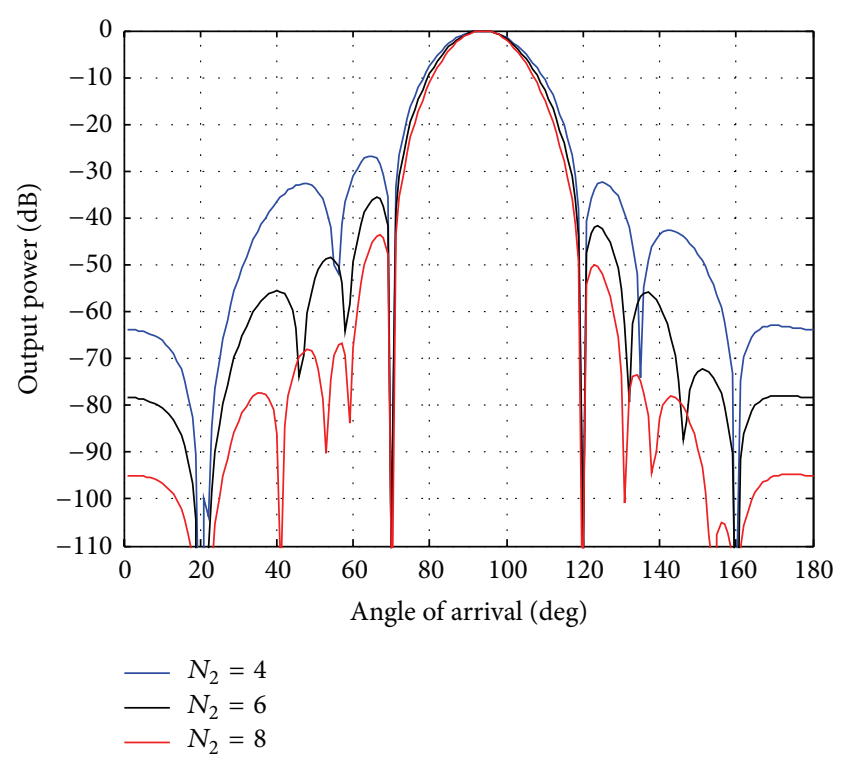

Figure 6: Performance of proposed algorithm for $N_{2}=4, N_{2}=6$, and $N_{2}=8$.

complex weights, $0.5 N_{1}\left(N_{1}+2 N_{2}-1\right)+1$ adders, and $N_{2}-1$ nulls.

The coefficients for the sidelobe suppression are not affected by the change in position of one or more nulls with the condition that the main beam does not change its direction. On the other hand if the main beam changes its position, the whole set of weights of the structure along with the progressive phase shift will be changed. The sidelobes can be suppressed more by increasing the number of coefficients dedicated for this purpose.

In future one can use the same approach for two- and three-dimensional arrays.

\section{Conflict of Interests}

All the authors of this paper declare that there is no conflict of interests regarding the publication of this paper.

\section{References}

[1] B. Friedlander and B. Porat, "Performance analysis of a nullsteering algorithm based on direction-of-arrival estimation," IEEE Transactions on Acoustics, Speech, and Signal Processing, vol. v, pp. 461-466, 1992.

[2] T. B. Vu, "Method of null steering without using phase shifters," IEE Proceedings H: Microwaves Optics and Antennas, vol. 131, no. 4, pp. 242-246, 1984.

[3] H. M. Ibrahim, "Null steering by real-weight control-a method of decoupling the weights," IEEE Transactions on Antennas and Propagation, vol. 39, no. 11, pp. 1648-1650, 1991.

[4] H. Steyskal, "Simple method for pattern nulling by phase perturbation," IEEE Transactions on Antennas and Propagation, vol. 31, no. 1, pp. 163-166, 1983.

[5] M. Mouhamadou, P. Vaudon, and M. Rammal, "Smart antenna array patterns synthesis: null steering and multi-user beamforming by phase control," Progress in Electromagnetics Research, vol. 60, pp. 95-106, 2006.

[6] Z. U. Khan, A. Naveed, I. M. Qureshi, and F. Zaman, "Independent null steering by decoupling complex weights," IEICE Electronics Express, vol. 8, no. 13, pp. 1008-1013, 2011.

[7] J. A. Hejres, A. Peng, and J. Hijres, "Fast method for sidelobe nulling in a partially adaptive linear array using the elements positions," IEEE Antennas and Wireless Propagation Letters, vol. 6, pp. 332-335, 2007.

[8] J. A. Hejres, "Array pattern nulling using the elevations of selected elements of phased antenna arrays," in Proceedings of the IEEE Antennas and Propagation Society International Symposium, vol. 2, pp. 68-71, July 2005.

[9] J. A. Hejres, "Null steering in phased arrays by controlling the positions of selected elements," IEEE Transactions on Antennas and Propagation, vol. 52, no. 11, pp. 2891-2895, 2004.

[10] F. Tokan and F. Güneş, "Interference suppression by optimising the positions of selected elements using generalised pattern search algorithm," IET Microwaves, Antennas and Propagation, vol. 5, no. 2, pp. 127-135, 2011.

[11] A. F. Morabito, A. Massa, P. Rocca, and T. Isernia, "An effective approach to the synthesis of phase-only reconfigurable linear arrays," IEEE Transactions on Antennas and Propagation, vol. 60, no. 8, pp. 3622-3631, 2012.

[12] J. Liu, A. B. Gershman, Z.-Q. Luo, and K. M. Wong, "Adaptive beamforming with sidelobe control: a second-order cone programming approach," IEEE Signal Processing Letters, vol. 10, no. 11, pp. 331-334, 2003.

[13] Y. Zhang, B. P. Ng, and Q. Wan, "Sidelobe suppression for adaptive beamforming with sparse constraint on beam pattern," Electronics Letters, vol. 44, no. 10, pp. 615-616, 2008.

[14] S. A. Schelkunoff, "A mathematical theory of linear arrays," The Bell System Technical Journal, vol. 22, pp. 80-107, 1943.

[15] S. Boyd and L. Vandenberghe, Convex Optimization, Cambridge University Press, 2004.

[16] M. Grant and S. Boyd, "CVX: a system for disciplined convex programming: cvx version 1.21,” 2011. 

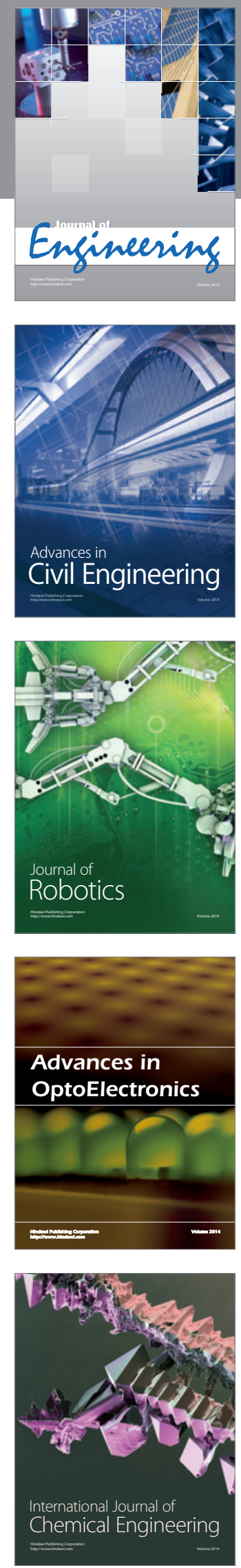

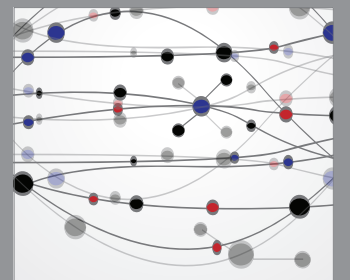

The Scientific World Journal
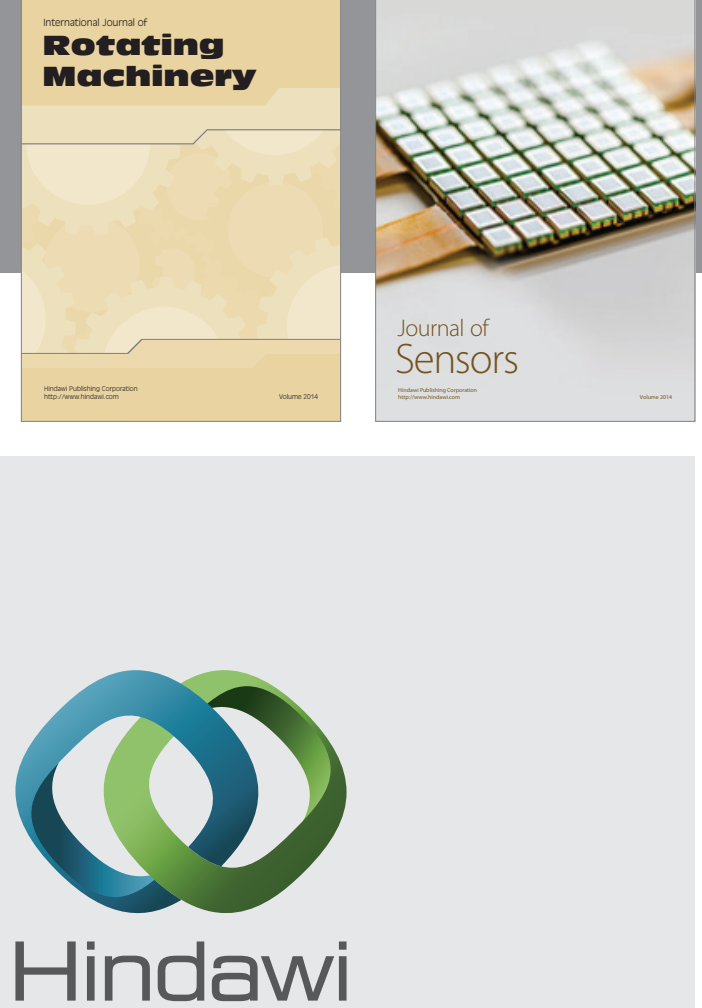

Submit your manuscripts at http://www.hindawi.com
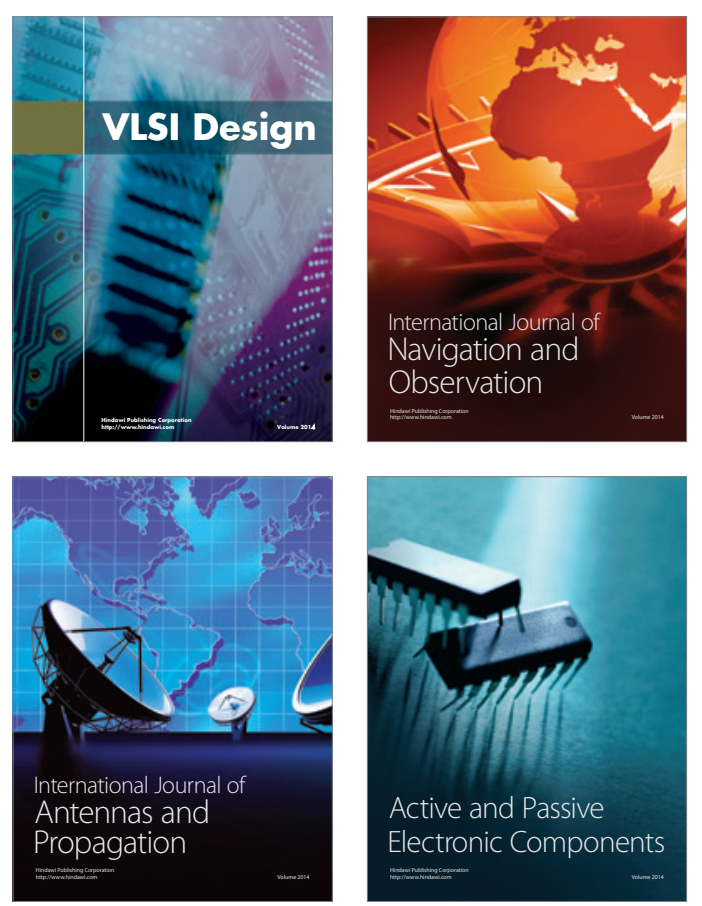
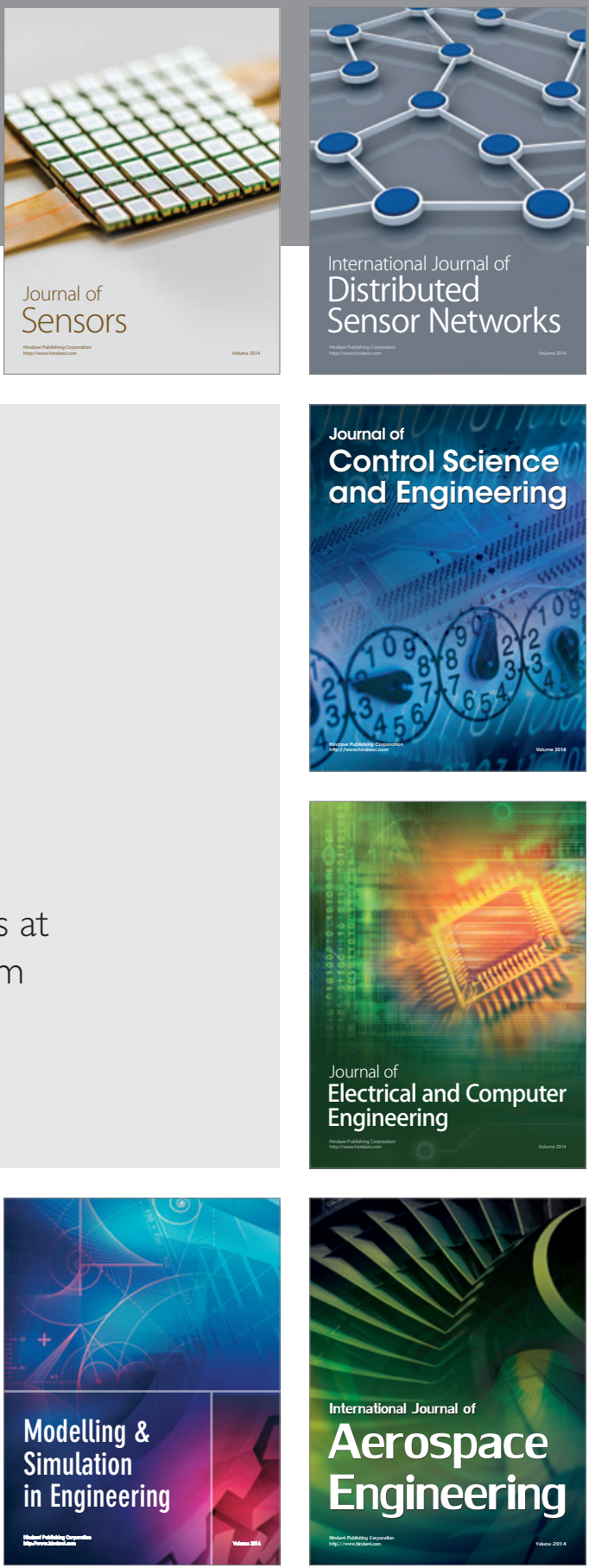

Journal of

Control Science

and Engineering
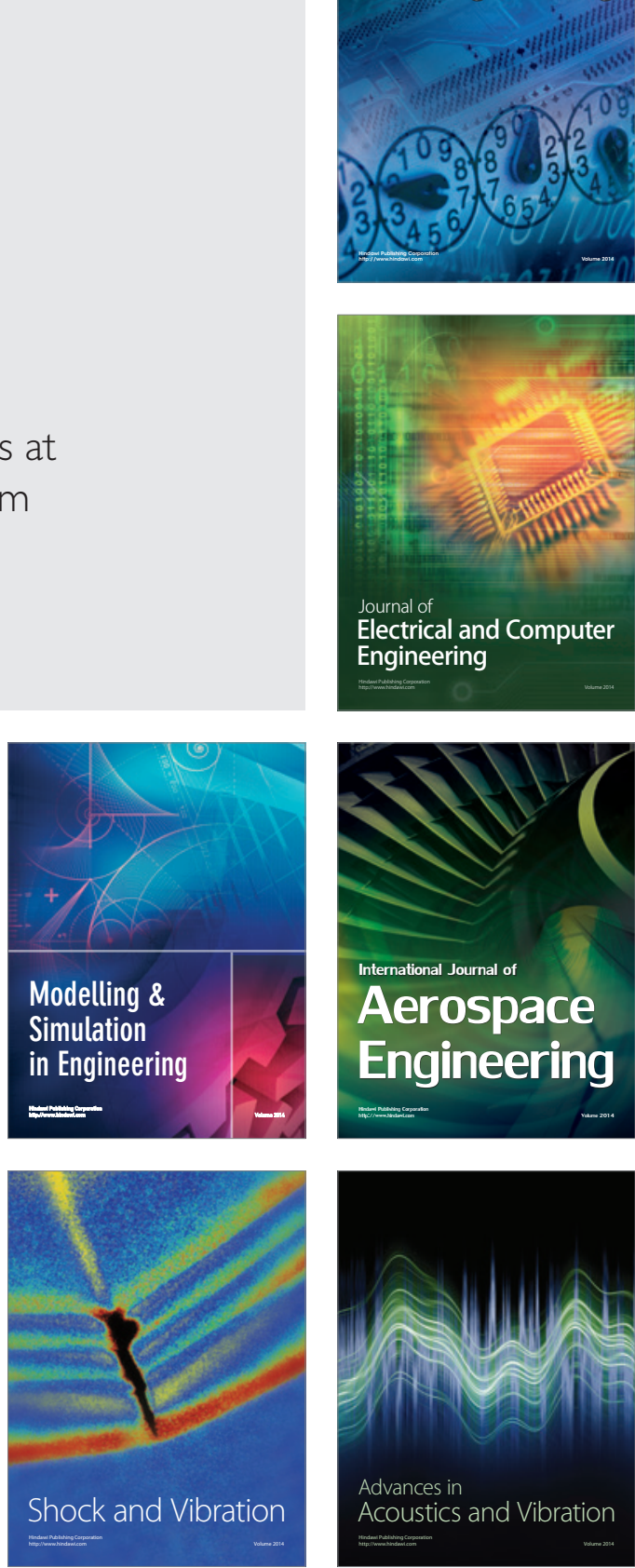